\title{
THE ROLE OF NUTRITIONAL HABITS AND BEHAVIOR FOR THE ETIOLOGY OF OVERWEIGHT AND OBESITY
}

\author{
T. Cvetanov* \\ Faculty of Medicine, Trakia University, Stara Zagora
}

\begin{abstract}
Obesity is one of the most widespread diseases of our days .Because of its wild distribution not only among adults but also among children, adolescents and young people, the WORLD HEALTH ORGANISATION (WHO) include it in the list of socially and medically significant diseases with serious effects on human health. The factors for its etiology and development are complex, but the most authors identify the unhealthy nutritional habits and lack of regular physical activity as leading ones.

The purpose of this study was to reveal the most common causes of weight increase above its normal levels in medical students.

Material and methods. The study included 120 students from the Medical Faculty of the Thracian university -Stara Zagora. They carried out an individual anonymous questionnaire, with a total of 21 questions - closed, open and mixed, which aim was to reveal some of the possible causes leading to excess weight in the contingent studied. Methods used: Research and analysis of information sources, survey sociological method, alternative analysis.

Results and discussion. In that report we will analyse the answers to those questions concerning the nutritional habits and behaviour of the respondents. It turns out that among the most common reasons for increasing body weight above the normal range is the presence of a number of unhealthy nutritional habits, such as the intake of high-energy food products of low biological value, the intake of a higher amount of food/overeating/and irregular eating.

Conclusion. Based on the research carried out, we can summarize that a large number of the students surveyed demonstrate a good knowledge about the healthy and balanced nutrition, but unfortunately most of them do not follow it because of their dynamic and strenuous daily life, and this in combination with the decreased habitual physical activity will inevitably lead to an increased body weight.
\end{abstract}

Key words: healthy and balanced nutrition, nutritional habits, overweight and obesity, students

\section{INTRODUCTION}

It is a well-known fact that obesity is a problem of extremely great medical-social importance. The World Health Organization (WHO) defines it as a "chronic disease", which is included in the INTERNATIONAL CLASSIFICATION, as "a disease with endocrine, nutritional, metabolic and immunological nature", with serious negative impact on the human health $(1,2)$. Its relevance is determined by the fact that it is a prerequisite for a number of serious diseases affecting the cardiovascular and respiratory systems (high blood pressure, heart failure, chronic obstructive pulmonary disease,

*Correspondence to: Todor Cvetanov, Faculty of Medicine - Trakia University - Stara Zagora bronchitis, asthma, etc.), musculoskeletal system (arthritis, arthroses), metabolic processes (diabetes, metabolic syndrome), frequently it becomes the cause for the development of some types of oncological diseases and various traumas. As a whole it affects human quality of life (3-5). Because of its wide distribution not only among adults but also among children, adolescents and young people, it is considered to be the modern epidemic in the world (6-9).

The factors for its etiology and development are complex and can be differentiated into two groups: endogenous - genetics (heredity). They are conservative and don't change as a result of external influences. The other group exogenous, includes social factors (family, residence, professional environment, emotional 
and psychological factors, as stress is one of the main one, some diseases, intake of certain medications, etc.). But most authors define unhealthy nutritional behaviour and habits and lack of enough habitual physical activity, as most important (10, 11, 1, 12-15).

THE PURPOSE of this study was to reveal the most common causes of body weight increase above the normal levels in medical students.

\section{MATERIAL AND METHODS}

The study included 120 students from the Medical Faculty of the Thracian universityStara Zagora. They carried out an individual anonymous questionnaire, with a total of 21 closed, mixed and open questions, which were aimed at detecting the physical and nutritional habits of the respondents, the reasons leading to an increase in body weight above the normal limits, the objectives and the reasons for taking part in a complex program for its correction.

In this paper we will analyse the answers to the questions concerning the nutritional behaviour and habits of the contingent researched.

Methods used: Research and analysis of information sources, survey sociological method, alternative analysis.

\section{RESULTS AND DISCUSSION}

The nutritional habits of people have a complex character and are determined by different factors. Some of them have subjective character (individual preferences, attitude to food, taste peculiarities, appetite, etc.) and others are social, such as professional engagement, financial opportunities, residence, social status etc. $(16,14)$.

The lifestyle of modern man is dynamic, strenuous, stressful, and very often physically inactive, and this is a serious prerequisite for the appearance of a number of harmful nutritional habits, which are one of the main factors for increasing morbidity and mortality (4) . It is an indisputable fact that as a result of the higher amount of calories intake with food, in comparison with the calories needed for the realization of the main metabolism and daily activities, the weight increases its values and often overcome the values of the norms for a given age, gender, height, physical activity $(10,11,1)$.

We were interested in whether the nutritional habits and behaviour played an essential role in the etiology of overweight and obesity in our investigated individuals.

To the question: What foods and drinks do you prefer? (Figure 1) the largest number of the respondents give their preference to fruits, vegetables and wholegrain foods (61\%). Unfortunately, those who prefer the sugar products, such as chocolate, cakes, ice cream are nearly half of the participants in the survey $(48 \%)$. Nearly half of the students (46\%) choose products like pizzas, pastas, burgers, etc. $41 \%$ like to drink carbonated and energizing drinks and alcohol. Milk and dairy products were preferred by $35 \%$ of respondents. Similar percentage of the respondents prefer meat, fish, eggs on one hand and nuts and pulses on the another $15 \%$. The fats and fat products have the least admirers $-8 \%$.

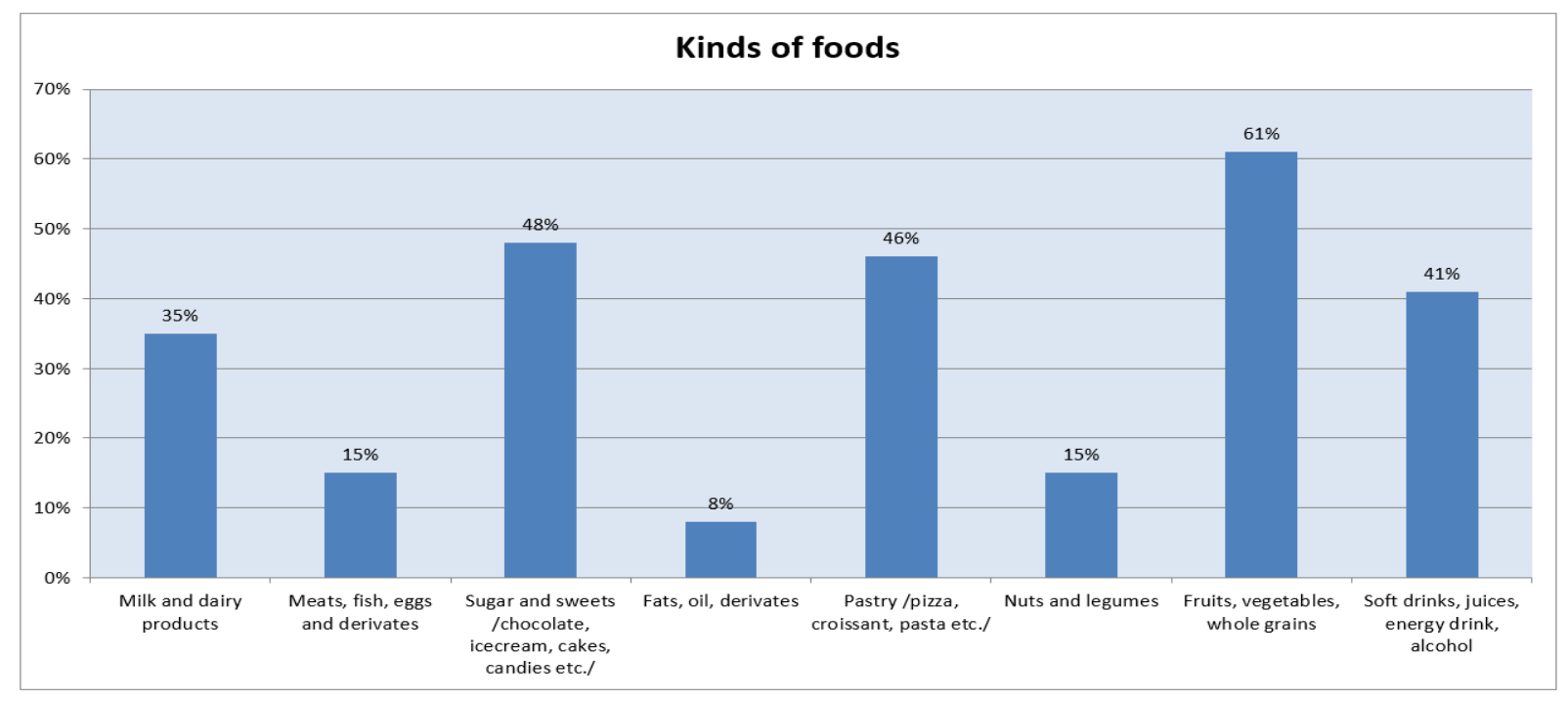

Figure 1. Preferred foods and drinks 
Obviously, among the useful complex carbohydrates (fruits, vegetables and whole grains, which are sources of energy, vitamins, minerals, fibres, and other health-beneficial compounds), in the daily diet of the respondents abound concentrated carbohydrate foods (mono and polysaccharides), carbonated and energy drinks and alcohol, which because of their high calorific value, systematically used lead to an increase of body weight. (3, 17, 18).

It is a positive fact that $1 / 3$ of the students prefer milk and milk products which are source of potassium, proteins, vitamins, minerals, fats which are very caloric! In that case, the consumption of products of low fat content should be recommended! $(18,15,2)$.

The complete proteins of both animal and vegetable origin are preferred by $15 \%$ of the studied girls. The last are basic building sources in the human body. They content all the amino acids, valuable minerals and vitamins, but also a lot of fats, therefore one should avoid some of them or take less quality and not so often and in limited quantities (7, $10,19)$.

The fats of plant and animal origin are of an important role for the functioning of the organism but at the same time they have an extreme caloric value, therefore, consumption, especially those of animal origin, should be avoided. It is not accidental that preference to this kind of food gave only $8 \%$ of respondents. From the analysis of the answers to this question it becomes clear that the interviewed students are familiar with the pyramid of healthy nutrition.

However their knowledge of healthy and harmful foods, students demonstrate unhealthy nutrition habits. This is most likely due to the dynamic and strenuous students' life and the lack of enough spare time needed to prepare healthy, balanced and varied food.

This statement is confirmed also by the answers to the question "What is the ratio between the main nutrients-proteins: carbohydrates: fats so that the nutrition can be rational, balanced and healthy?" (Figure 2). A large number of the respondents $(75 \%)$, gave answer B - which is the correct one (15: 55 : 15). Responses $A$ and $C$ have been given by $10 \%$. Only $5 \%$ can't answer that question. These results are not surprising, having in mind that the respondents are medical students and it is logical to have a high health culture and knowledge, compared, for example, with students from other Universities. Here, however, there is a contradiction between the knowledge and the actual behaviour (according to the answers of the previous question).

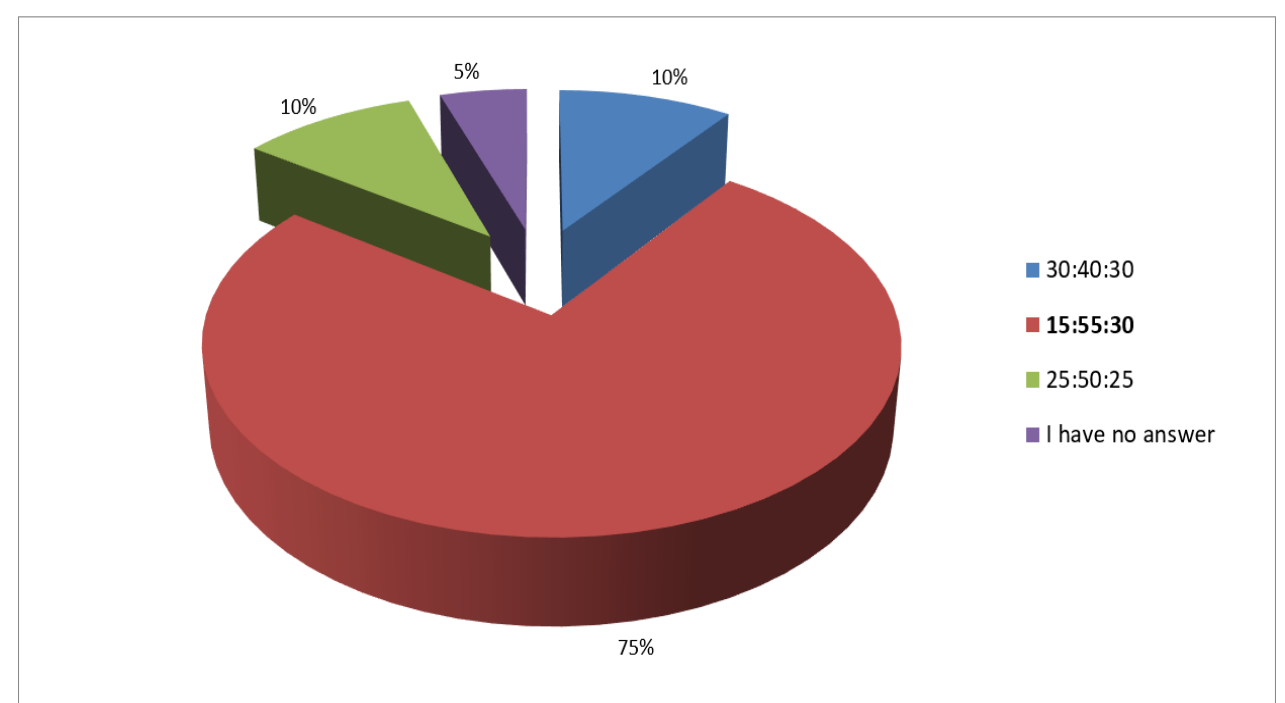

Figure 2. Ratio between the main nutrients-proteins: carbohydrates: fats so that the nutrition can be rational, balanced and healthy

The food quantity is also of a great importance besides its quality and composition, although the information about this factor is not always accurate, as many overweight people are not honest enough and conceals the truth about the amount of food they consumed $(6,8,17,1)$.

Systemic overeating will inevitably lead to an increase in body weight and, respectively, in 
fat tissue. In this regard, we asked a question about the frequency of food intake (Figure 3). Most of the respondents eat one or two times a day ( 25 and $45 \%)$. Three times a day eat $16 \%$ of the surveyed, 4 times $-9 \%$ and only $5 \%$ eat more than 4 times. In a similar diet, the intake of a larger quantity of food is larger in order to satisfy the hunger $(6,9,20,13)$.

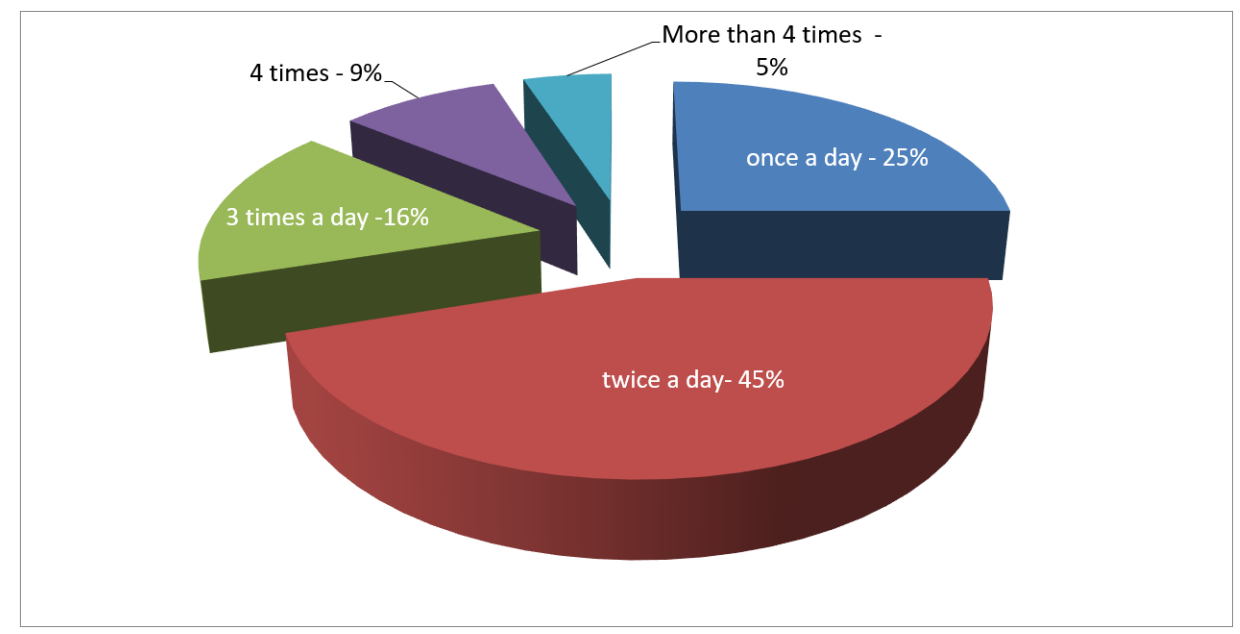

Figure 3. Frequency of food intake

Most of the authors and the practice prove that five-meal with a small, low-calorie and varied diet is one of the prerequisites for a healthy weight reduction or for maintaining it within normal limits.

And as we found that $70 \%$ of the respondents eat $1-2$ times a day, we decided to find out at what time of the day is the main meal. In this regard, we asked the question "At what time of the day do you have your meal? (Figure 4).
The answers were not surprising. 54\% of the girls said that the evening meal is primary, $26 \%$ of them set lunch as a main meal and only $15 \%$ said it was the breakfast. The remaining percentages are divided between the second morning and afternoon snacks. (for those who eat 5 times a day). It has been experimentally established that the intake of a large amount of food at night is unhealthy and results in excess weight $(6,8,16)$.

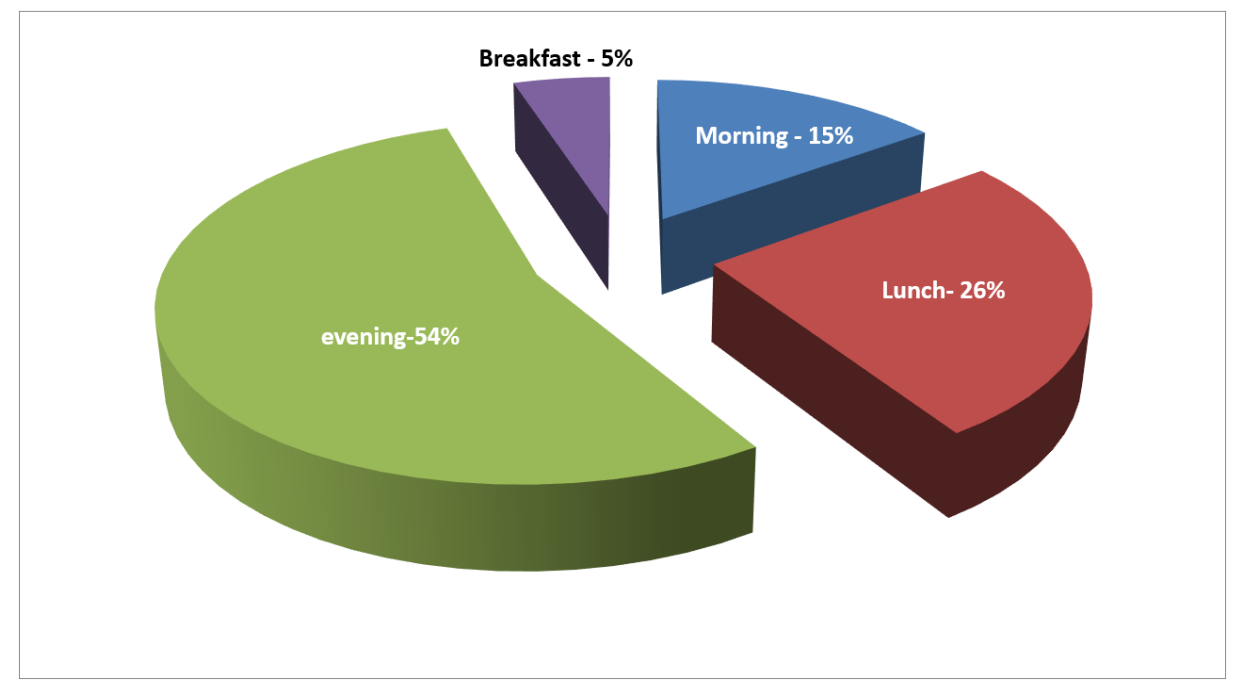

Figure 4. Timing of food intake

Many authors consider that the emotional and mental state very often becomes the cause of greater food intake and especially when this is repeated systematically, will inevitably lead to an increase in body weight $(6,8,21,22,23)$. The answers to the question "Under what circumstances do you consume more food than necessary (overeat)?" (Figure 5) support that thesis. The highest percentage has the answer "when I'm sad, lonely, depressed, stressed out, angry"-48\%, followed by the answer "'when I'm happy, cheerful, joyful and successful "$35 \% .28 \%$ answered that they consume much more food when they eat with friends in a pub. 
A similar percentage also has the answer "when I eat in a fast food restaurant" - 32\%. Respondents receive the most - little food when they eat at home $-23 \%$. Obviously, the place to eat, the company and the emotional state, whether positive or negative, have a significant impact on the amount of food taken. As a whole people eat adequately and healthy when prepare their own food following the healthier principles and consuming it at home! (23-27).

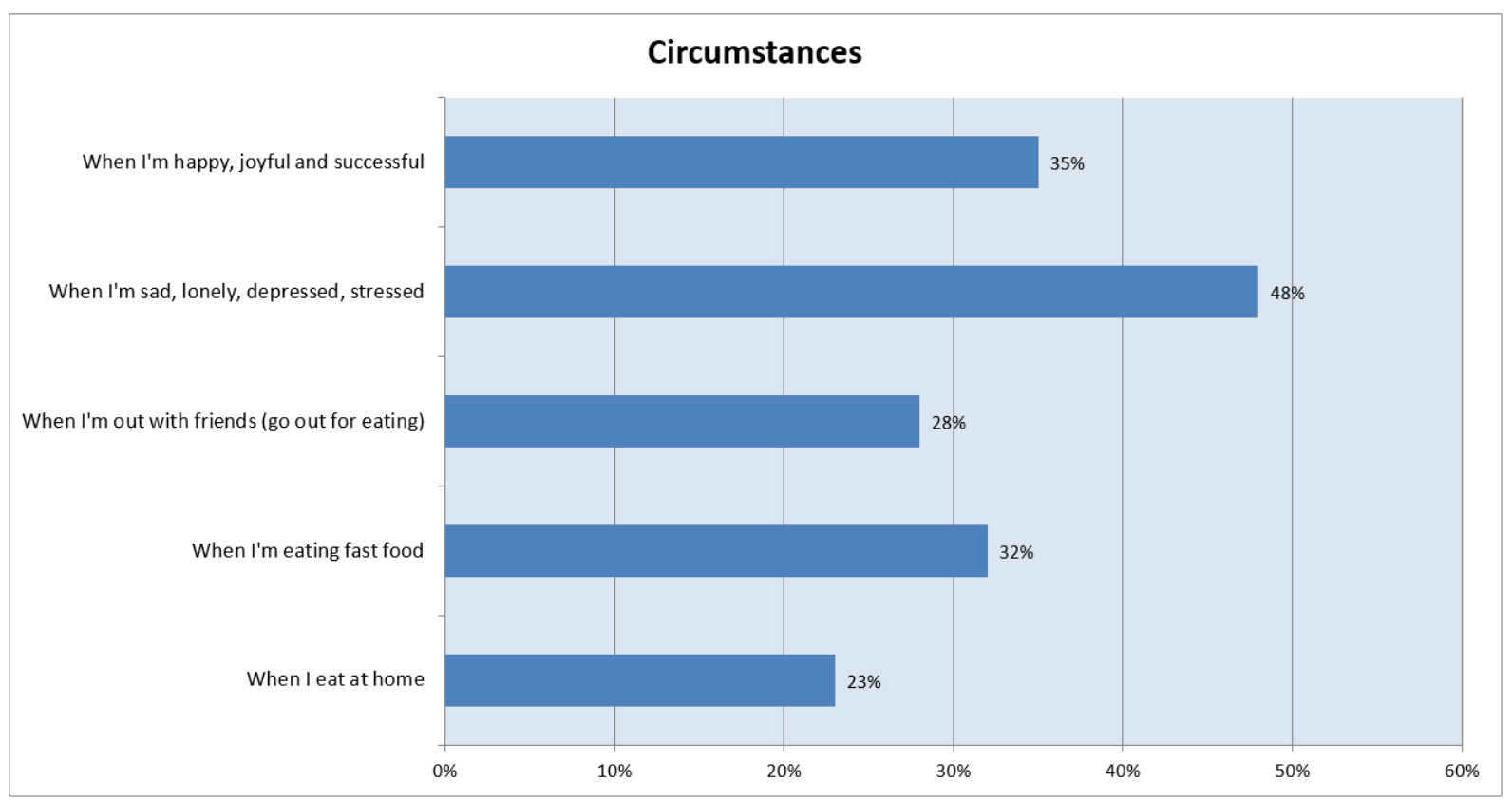

Figure 5. Circumstances for consuming more food than necessary (overeat)

From the analysis of the responses from the survey, we can CONCLUDE that the majority of the students surveyed have a good health culture and knowledge of adequate and balanced nutrition, but unfortunately there is a contradiction between knowledge and nutritional behaviour and habits.

The most common reasons for increasing of body weight above the normal levels is the intake of high-energy food products of low biological value, the intake of a higher quantity of food (overeating) and the irregular eating.

\section{REFERENCES}

1. Gattceva P. et al. Relationship between eating habits and overweight in adolescence. 12, N 4, p. 33-41.2009.

2. WHO Methods and development. In: Child Growth Standards, WHO, Geneva, 2006.

3. Balabansky L. Nutrition and dietetics. Med and fizk., S.,1988.

4. Barensky L. Overweight, obesity and cardiovascular risk. Nauka endokrinologiia, 7, No 1, p. 9-18, 2013.

5. Bačchevanov St. Health Diets. Millennium, S. 2012.

6. Asenova R. Longitudinal study of the prevalence of excess weight at early school age by sex and age Suvr. med., 57, No 6, p. 3-7, 2006.

7. Baikova D. The obesity problem in Bulgaria. Health Care, issue 1, 2004.

8. Consul P. et al. Prevalence, social and family risk factors for the development of overweight and obesity in students aged 1619 years in Plovdiv. Nauka endokrinologiia, 10, N 4, p. 131-136, 2016

9. Dimitrova D. Physical activity and health. Avanguard Prima, S., 2014.

10.Baikova D." Let's lose weight without starvation. "Sun ", S., 2013.

11.Crovetto M. et al. Effect of healthy and unhealthy habits on obesity: a multicentric study. Nutrition. 54:7-11,2018

12.Georgiev G., K. Casakova.Epidemiology and consequences of overweight and obesity Obshta med., 16, N 1, p. 48-56, 2014.

13.Hootman K. et al. Stress and psychological constructs related to eating behaviour are associated with anthropometry and body composition in young adults. Appetite, 125, 287-294, 2018.

14. Sharkley B. Fitness and Health. Human Kinetics., 199.

15.WHO Report of a joint WHO/FAO Expert Consultation. Diet, Nutrition and 
Prevention of Chronic Diseases. TRS 916, WHO, Geneva, 2002.

16. Dimitrova D. Obesity and diettherapy. Avanguard Prima, S.,2012.

17.Dimitrova D. Physical activity and health. Avanguard Prima, S.,2014.

18. Stefanova P. Positive health behaviour of students - a condition for the prevention of obesity. Int.J.KNOWLEDGE, vol.26, 4, Skopje, 201.

19.Baikova D. NEWS, No. 3., 200 .

20.Jiang Y et al. Association between TakeOut Food Consumption and Obesity among Chinese University Students: A CrossSectional Study. Journal of Adolescent Health.

21.Kiryakova St. Nutritional behaviour of students, such as prevention of chronic noninfectious diseases. Science Dietetics, No.3., 2011.

22. Baikova D. Body mass index in young women in Bulgaria - integral indicator of their anthropometric and nutritional status. Science Diet, No.3., 2012.

23. Borisova A.M. aet al. Study on the prevalence of obesity in the Bulgarian population. Endocrinology, No2, 2015.

24.Paskaleva R. Kinesitherapy in common diseases in childhood and relaxation techniques. Textbook. Publisher: EXPRESS Gabrovo; 227s, 2015.

25.Paskaleva R. Kinesitherapy and art therapy for diseases in childhood. Textbook. Publisher: EX-PRESS Gabrovo; 180 s, 2020.

26.Petrova S. et al. Monitoring of the nutritional status of the population in Bulgaria. Science Dietetics, 2-4 .

27.Gorneska A. Modern challenges of excess weight and insufficient physical activity Meditsinski meridiani, 5, N 2, p. 63-67, 2015.

28. Study on the prevalence of obesity in the Bulgarian population in 2012. Endocrinology, 2015, issue 2. 